\title{
Comprehensive serum and tissue microRNA profiling in dedifferentiated liposarcoma
}

\author{
ISAKU KOHAMA $^{1,2}$, NAOFUMI ASANO ${ }^{3}$, JUNTARO MATSUZAKI ${ }^{1}$, \\ YUSUKE YAMAMOTO $^{1}$, TOMOFUMI YAMAMOTO ${ }^{1}$, RYOU-U TAKAHASHI $^{4}$, \\ EISUKE KOBAYASHI ${ }^{5}$, SATOKO TAKIZAWA ${ }^{6}$, HIROMI SAKAMOTO ${ }^{7}$, KEN KATO $^{8}$, \\ HIROYUKI FUJIMOTO ${ }^{9}$, HIROTAKA CHIKUDA ${ }^{2}$, AKIRA KAWAI $^{5}$ and TAKAHIRO OCHIYA ${ }^{1,10}$ \\ ${ }^{1}$ Division of Molecular and Cellular Medicine, National Cancer Center Research Institute, Tokyo 104-0045; \\ ${ }^{2}$ Department of Orthopedic Surgery, Gunma University Graduate School of Medicine, Maebashi, Gunma 371-8511; \\ ${ }^{3}$ Department of Orthopedic Surgery, Keio University School of Medicine, Tokyo 160-8582; ${ }^{4}$ Department of Cellular and \\ Molecular Biology, Division of Integrated Medical Science, Graduate School of Biomedical Sciences, Hiroshima \\ University, Hiroshima 734-8553; ${ }^{5}$ Department of Musculoskeletal Oncology, National Cancer Center Hospital, \\ Tokyo 104-0045; ${ }^{6}$ New Frontiers Research Institute, Toray Industries, Kamakura, Kanagawa 247-8555; \\ ${ }^{7}$ Department of Clinical Genomics, Fundamental Innovative Oncology Core, National Cancer Center Research Institute; \\ Departments of ${ }^{8}$ Gastrointestinal Medical Oncology and ${ }^{9}$ Urology, National Cancer Center Hospital, \\ Tokyo 104-0045; ${ }^{10}$ Department of Molecular and Cellular Medicine, Institute of Medical Science, Tokyo \\ Medical University, Tokyo 160-0023, Japan
}

Received May 22, 2020; Accepted April 30, 2021

DOI: $10.3892 / 01.2021 .12884$

\begin{abstract}
Sarcoma is a rare cancer with several subtypes; therefore, our understanding of the pathogenesis of sarcoma is limited, and designing effective treatments is difficult. Circulating microRNAs (miRNAs), including exosomal miRNAs, have attracted attention as biomarkers in cancer. However, the roles of miRNAs and exosomes in sarcoma remain unclear. The present analysis of tissue and serum miRNA expression in osteosarcoma, Ewing's sarcoma and dedifferentiated liposarcoma (DDLPS) identified miR-1246, $-4532,-4454,-619-5 p$ and -6126 as biomarkers for DDLPS. These miRNAs were highly expressed in human DDLPS cell lines and exosomes, suggesting that they are secreted from DDLPS tissues. The present results suggested that specific miRNAs may be used as biomarkers for early diagnosis or treatment targets in DDLPS.
\end{abstract}

Correspondence to: Professor Takahiro Ochiya, Department of Molecular and Cellular Medicine, Institute of Medical Science, Tokyo Medical University, 6-7-1 Nishi-Shinjuku, Shinjuku-ku, Tokyo 160-0023, Japan

E-mail: tochiya@tokyo-med.ac.jp

Key words: sarcoma, cancer, microRNA, exosome, liquid biopsy

\section{Introduction}

Sarcoma has numerous subtypes and typically develops at a relatively young age compared with other cancers (1). Osteosarcoma (OS), the most common primary sarcoma of bone, preferentially develops at 10-14 years of age, which is a period of accelerated bone growth $(2,3)$. Ewing's sarcoma (EWS) is a small round sarcoma and the second most common sarcoma of bone in children (1). Liposarcoma (LPS), a common subtype of soft tissue sarcoma (STS), accounts for $20 \%$ of STS cases (4). LPS is classified into 4 categories, which are well differentiated liposarcoma (WDLPS), dedifferentiated liposarcoma (DDLPS), myxoid liposarcoma and pleomorphic liposarcoma $(1,4)$. DDLPS accounts for $\sim 20 \%$ of these and shares a genetic background with WDLPS and occurs focally in the WDLPS lesion $(1,5)$.

microRNAs (miRNAs/miRs) are non-coding RNAs with a length of 17-25 base pairs. miRNAs regulate gene expression at the transcriptional level by binding to target mRNAs (6). Over a period of 10 years of miRNA research, numerous functions of miRNAs have been identified, including their involvement in cancer (7). Exosomes are small vesicles of $\sim 100 \mathrm{~nm}$ in size that function as an active transport system in cells (8). Exosomes are enclosed in a lipid bilayer membrane and incorporate mRNAs, miRNAs and proteins (8). Exosomes are released from most cell types and function as intercellular communication tools (8). Cancer cells interact with the microenvironment through exosomes, and tumor-derived exosomes induce premetastatic niches (9).

There are currently no effective biomarkers for sarcomas, to the best of our knowledge. In recent years, circulating miRNAs 
have attracted attention as candidate biomarkers in several cancers, and these miRNAs are released into the extracellular space by small vesicles, particularly exosomes (7).

Several circulating miRNAs have been identified as candidate markers for OS. The expression levels of miR-195,-Let7A, -9 and -21 are increased in the serum or plasma of patients with OS compared with healthy controls (10-13). miR-135b, -148a, $-27 \mathrm{a}$ and -9 levels are increased in the exosomes of the serum of patients with OS with a good response to chemotherapy, and these miRNAs can predict the therapeutic efficacy (14).

DDLPS is difficult to detect because it often occurs in the retroperitoneum and trunk $(1,15,16)$. Therefore, it is often discovered after it has grown and is often difficult to resect. When comparing miRNAs in formalin-fixed paraffin-embedded LPS and adipose tissue samples, miR-155 and -21 are upregulated in LPS samples $(17,18)$. High expression of miR-155 and -26a-2 is correlated with a poor prognosis in DDLPS $(18,19)$. miR-25-3p and $-92 a-3 p$ are highly expressed in peripheral blood plasma vesicles derived from human LPS patient samples. These miRNAs are secreted from LPS cell lines through extracellular vesicles and transferred to macrophages (20).

In previous work from our group, it was identified that serum miRNAs that can serve as biomarkers for certain types of cancer, such as sarcoma, bladder cancer and ovarian cancer (21-23). However, whether the identified miRNAs are released from the tumor site remains unclear. In the present study, the relevance of the identified miRNAs to sarcoma was determined by comparing miRNA expression levels between serum and tissue samples from the same patient based on the microarray data.

\section{Materials and methods}

Clinical samples. All clinical serum samples were obtained from patients undergoing tumor resection at the National Cancer Center Hospital (Tokyo, Japan) ( $\mathrm{NCCH}$ ) between January 2007 and December 2013. The patients that became inoperable during preoperative treatment and were judged to be inappropriate as participants by the doctor or refused to participate in this study were excluded. The clinicopathological data were collected and TNM staging was performed according to French Fédération Nationale des Centres de Lutte Contre Le Cancer (FNCLCC) system described in American Joint Committee on Cancer (AJCC) system, 7 th edition (1). Serum samples were stored at $4^{\circ} \mathrm{C}$ for 1 week and then stored at $-20^{\circ} \mathrm{C}$ until further use. The serum samples were collected before the operation or preoperative treatment at the $\mathrm{NCCH}$. Tissue samples were obtained from surgical specimens of patients undergoing surgery at the $\mathrm{NCCH}$ and stored at $-80^{\circ} \mathrm{C}$ in the NCC Biobank. The study included 22 OS samples, 17 DDLPS samples and three EWS samples (Tables SI-III). All of these samples were used by Asano et al (21). The present study was approved by the NCCH Institutional Review Board (Tokyo, Japan; approval nos. 2004-050, 2013-111 and 2015-266). Written informed consent was obtained from each participant on the first visit. When the patient was under 20 years old, the informed consent was obtained from their parents, relatives or guardians of minors.
miRNA expression array of clinical serum and tissue samples. Serum RNA was extracted from $300 \mu \mathrm{l}$ of serum using the $3 \mathrm{D}-G e n \mathrm{e}^{\circledR}$ RNA extraction reagent (Toray Industries, Inc.). Fresh-frozen tissues were crushed to a powder using a Multibead Shocker (Yasui Kikai Corporation) under liquid nitrogen $\left(-196^{\circ} \mathrm{C}\right)$. Total RNA was extracted from frozen tumor tissue powder using the miRNeasy Mini kit (cat. no. 217004; Qiagen $\mathrm{GmbH}$ ). Comprehensive miRNA expression analysis was performed using the 3D-Gene ${ }^{\circledR}$ miRNA Labeling kit and the 3D-Gene ${ }^{\circledR}$ Human miRNA Oligo Chip (both Toray Industries, Inc.), which was designed to detect 2,588 miRNA sequences registered in miRBase release 21 database (http://www.mirbase.org/). Microarray experiments were performed by Kamakura Techno-Science Inc. miRNAs with a signal intensity $>2^{6}$ were considered detected miRNAs. Principal component analysis (PCA) map and heatmap were generated by Genomics Suite version 6.6 (Partek Inc.).

Cell culture. Human DDLPS cell lines (LP6 and LPS12) were previously established and kindly provided by Dr Andrew J. Wagner (Dana Farber Cancer Institute; USA). Human adipose-derived stem cells (ADSCs) were purchased from Invitrogen; Thermo Fisher Scientific, Inc. (cat. R7788115). LP6 cells were cultured in RPMI-1640 medium (cat. 11875093; Thermo Fisher Scientific, Inc.) supplemented with $10 \%$ fetal bovine serum (FBS; cat. 10270-106; Thermo Fisher Scientific, Inc.). LPS12 cells were cultured in DMEM/F12 (cat. 21331020; Thermo Fisher Scientific, Inc.) supplemented with $10 \%$ FBS and GlutaMAX (cat. 35050061; Thermo Fisher Scientific, Inc.). ADSCs were cultured in MesenPRO RS ${ }^{\mathrm{TM}}$ medium (cat. 12746012; Thermo Fisher Scientific, Inc.). All cell cultures were treated with $1 \%$ antibiotic-antimycotic solution (cat. 15240062; Thermo Fisher Scientific, Inc.) and cultured at $37^{\circ} \mathrm{C}$ in $5 \% \mathrm{CO}_{2}$.

Cell proliferation assay. LP6 cells were seeded at a density of $5 \times 10^{3}$ cells per well into a 96 -well dish at $37^{\circ} \mathrm{C}$ in $5 \% \mathrm{CO}_{2}$. After 1 day of seeding, the medium was replaced by miRNA mimics (hsa-miR-1246, ID MC13182; 5'AAUGGAUUU UUGGAGCAGG-3'; hsa-miR-4532, ID MC21908; 5'-CCC CGGGGAGCCCGGCG-3'; hsa-miR-4454, ID: MC21186; 5'-GGAUCCGAGUCACGGCACCA-3'; hsa-miR-619-5p, ID: MC28761; 5'-GCUGGGAUUACAGGCAUGAGCC-3'; and hsa-miR-6126, ID: MCMC25200; 5'-GUGAAGGCC CGGCGGAGA-3'; all Thermo Fisher Scientific, Inc.) in DharmaFECT reagent-1 (cat. no. T-2001-02; GE Healthcare Dharmacon, Inc.) for miRNA transfection. Negative Control \#1 (NC) (cat. no. 4464058; Thermo Fisher Scientific, Inc.) was used as a non-targeting negative control. The concentrations of miRNA mimics and NC were $2 \mu \mathrm{M}$ in the original stocks. The transfection efficiency was estimated $(n=4)$ based the average using HiLyte Fluor488 negative control miRNA mimic (Nippon Gene Co., Ltd.) and evaluated using reverse transcriptionquantitative PCR with miRNA-specific primers. Each miRNA was added to three wells. For all miRNA mimic transfection, final concentrations were $10 \mathrm{nM}$. The time of transfection was $8 \mathrm{~h}$ at $37^{\circ} \mathrm{C}$ in $5 \% \mathrm{CO}_{2}$. After transfection $(24,48$ and $72 \mathrm{~h})$, the number of living cells was counted using the Cell Counting Kit- 8 (Dojindo Molecular Technologies, Inc.) for 3 days, and measurements (Gen5 Synergy H4; BioTek Instruments, Inc.) 
were performed three times. The average value of day 2 and 3 was normalized to that of day 1 . Proliferation curves were drawn with the average value, and the standard deviation was calculated. Images were captured using a fluorescent microscope (BZ-X700; Keyence Corporation).

Purification and analysis of exosomes. Exosomes were prepared as previously described (24). Briefly, to avoid the contamination of exosomes from FBS, cells were washed with PBS and the culture medium was replaced with advanced RPMI medium (cat. 12633012; Thermo Fisher Scientific, Inc.) with 2 mM L-glutamine (cat. 25030149; Thermo Fisher Scientific, Inc.) for LP6 cells and advanced DMEM/F12 medium (cat. 12491015; Thermo Fisher Scientific, Inc.) with $2 \mathrm{mM}$ L-glutamine for LPS12 cells. The ADSC culture medium was replaced by StemPro ${ }^{\mathrm{TM}}$ MSC SFM medium (cat. A1033201; Thermo Fisher Scientific, Inc.). The LP6, LPS12 and ADSC cells were incubated for $48 \mathrm{~h}$ at $37^{\circ} \mathrm{C}$ in $5 \% \mathrm{CO}_{2}$. After $48 \mathrm{~h}$ of incubation, the conditioned medium (CM) was collected and centrifuged at $2,000 \mathrm{x}$ g for $10 \mathrm{~min}$ at $4^{\circ} \mathrm{C}$. The supernatant was filtered through a $0.22-\mu \mathrm{m}$ filter (EMD Millipore). The filtered CM was ultracentrifuged at 110,000 $\mathrm{x}$ g using a SW41Ti rotor for 70 min at $4^{\circ} \mathrm{C}$ using an Optima XPN-100 Ultracentrifuge (Beckman Coulter, Inc.). The supernatant was discarded, and the pellet was washed by adding PBS, ultracentrifuged at $35,000 \mathrm{rpm} 110,000 \mathrm{x} \mathrm{g}$ using the SW41Ti rotor for $70 \mathrm{~min}$ at $4^{\circ} \mathrm{C}$, and resuspended in PBS. For determination of the size distribution of exosomes, nanoparticle tracking analysis was performed using the NanoSight system (NanoSight; Malvern Panalytical, Ltd.) in samples diluted 500-1,000-fold with PBS for analysis (24).

RNA extraction and RT-qPCR. miRNAs were isolated from the LP6, LPS12 and ADSC cells and exosomes using the miRNeasy ${ }^{\circledR}$ Mini kit (cat. no. 217004; Qiagen GmbH), and cDNA was produced using the miScript II RT kit (cat. no. 218160; Qiagen GmbH) according to the manufacturer's instructions. The cDNA samples with miScript primers were subjected to quantitative PCR using a miScript ${ }^{\circledR}$ SYBR $^{\circledR}$ Green PCR kit (cat. no. 218075; Qiagen GmbH). Reactions were performed three times on the StepOnePlus Real-Time PCR system (Applied Biosystems; Thermo Fisher Scientific, Inc.). The following specific primers were used: Hs_miR-1246_1 (cat.no. MS00014224), Hs_miR-4532_1 (cat. no. MS00040705), Hs_miR-4454_1 (cat. no. MS00037597), Hs_miR-619-5p_1 (cat. no. MS00046032) and Hs miR-6126_1 (cat. no. MS00045416) (all Qiagen GmbH). The expression levels were normalized to those of RNU6-2_11 (cat. no. MS00033740; Qiagen $\mathrm{GmbH}$ ), and relative expression was calculated using the $2^{-\Delta \Delta \mathrm{Cq}}$ method (25). The expression levels of each miRNA in LP6 and LPS12 were compared with those in ADSCs to calculate the fold-changes. The average values and standard deviations were calculated.

Immunoblotting. In total, $1 \mu \mathrm{g}$ of exosomes were extracted by Sample Buffer Solution (2-Mercaptoethanol-) (FUJIFILM Wako Pure Chemical Corporation) and $1 \mu \mathrm{g}$ of proteins were loaded per lane onto 4-15\% Mini-PROTEAN TGX gels (Bio-Rad Laboratories, Inc.) and electrotransferred (100 V, $30 \mathrm{~mA}$ ) (26). The proteins were transferred to polyvinylidene difluoride membranes (EMD Millipore). The membranes were blocked with Blocking One solution (cat. 03953-95; Nacalai Tesque) on shacking machine for $1 \mathrm{~h}$ at room temperature and then incubated for $1 \mathrm{~h}$ at room temperature with primary antibodies: Anti-CD63 (1:1,000; cat. no. 12A12; Cosmo Bio Co., Ltd.) and anti-CD9 (1:1,000; cat. no. 8A12; Cosmo Bio Co., Ltd.). After washing, the membranes were incubated for $1 \mathrm{~h}$ at room temperature with secondary antibodies (horseradish peroxidase-linked anti-mouse IgG; cat. no. NA931 or horseradish peroxidase-linked anti-rabbit IgG; cat. no. Na934; both 1:5,000; GE Healthcare). After washing, the membranes were then exposed to ImmunoStar LD for development (cat. 292-69903; FUJIFILM Wako Pure Chemical Corporation).

Statistical analysis. miRNA expression levels were analyzed using Pearson's correlation, with the vertical axis representing tissues and the horizontal axis representing serum. The expression levels of miRNAs in cells and exosomes were compared by calculating the average values and the standard deviation. The data are presented as mean \pm SD of 3 or 4 biological replicates. A total of 3 biological replicates were performed for the experiments of miRNA extraction from cell lines and exosomes and cell proliferation. A total of 4 biological replicates were performed of the transfections of fluorescently-labeled miRNA mimic. The significance of the average values was analyzed using one-way ANOVA with a Tukey's HSD post hoc test. $\mathrm{P}<0.05$ was considered to indicate a statistically significant difference. Statistical analyses were performed using Excel 2019 (Microsoft Corporation) and online One-way ANOVA with post-hoc Tukey HSD Test Calculator for comparing multiple treatments (https://astatsa. com/OneWay_Anova_with_TukeyHSD/).

\section{Results}

Expression of miRNAs in the tissues and serum of patients with sarcoma. A total of 22 OS, 17 DDLPS and three EWS samples were analyzed. Overall, $830 \mathrm{miRNAs}$ were detected in tissues and 142 miRNAs in serum (Fig. 1A), and 141 miRNAs were expressed both in tissues and serum. Fig. 1B shows the results of hierarchical unsupervised clustering with miRNAs on the vertical axis and clinical cases on the horizontal axis. The expression of miRNAs was generally higher in tissues compared with in serum. Based on the PCA map (Fig. 1C), DDLPS and OS samples were separated in both serum and tissue samples. The expression profiles in tissues and serum were separated in the PCA map (Fig. 1C, right panel). Different trends were observed in miRNA expression patterns for each tumor type and miRNAs that were highly expressed in both tumor and serum miRNAs were found.

Correlation coefficients of sarcoma samples and DDLPS samples. The Pearson's correlation coefficients in miRNA expression between the serum and tissues were calculated (Fig. 2A). In the histograms of Fig. 2A, the distribution of Pearson correlation coefficient (R) was shown. In OS and DDLPS, there were a few miRNA which showed positive correlation between the serum and the tissues (Fig. 2A). Although miRNAs in EWS samples were expressed at high 
A
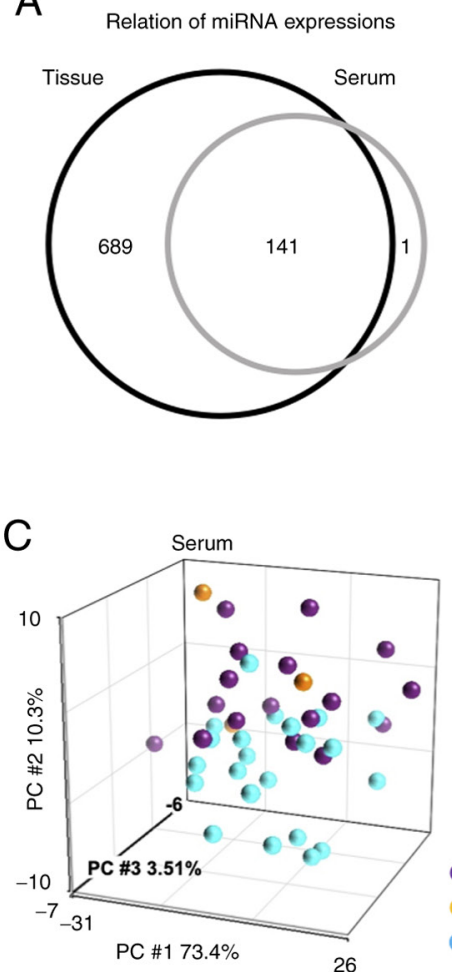

B

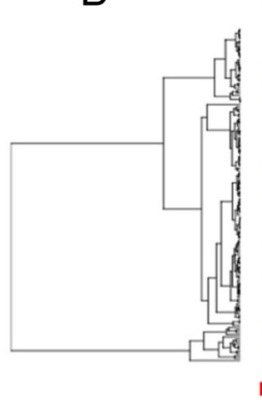

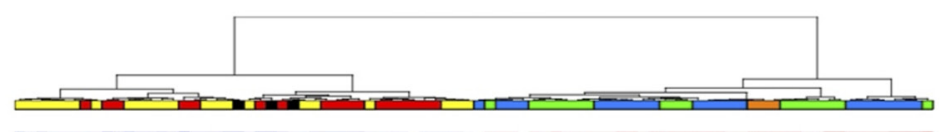

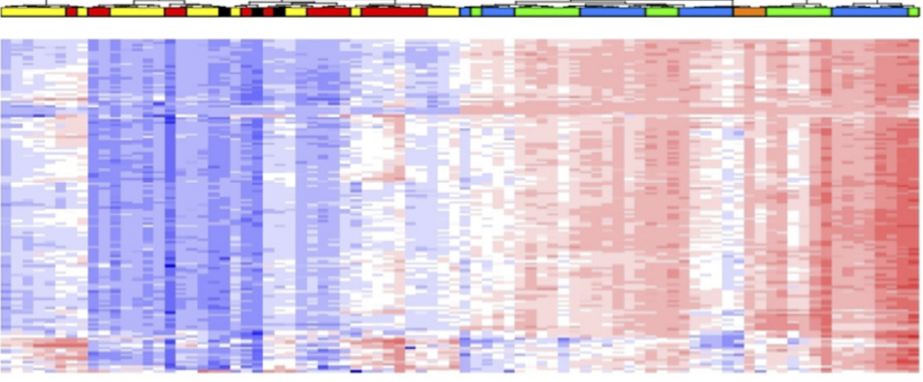

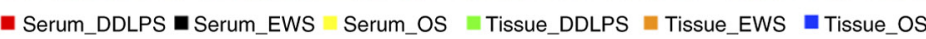

Figure 1. miRNA expression in the tissues and serum of patients with sarcoma. (A) Number of miRNAs with a signal intensity $>2^{6}$ in tissues and serum. (B) Heatmap of miRNAs in tissue and serum samples (the vertical axis represents miRNAs and the horizontal axis represents the serum and tissue samples for each subtype). (C) Principal component analysis map of each serum and tissue sample. miRNA, microRNA; DDLPS, dedifferentiated liposarcoma; EWS, Ewing's sarcoma; OS, osteosarcoma.

A
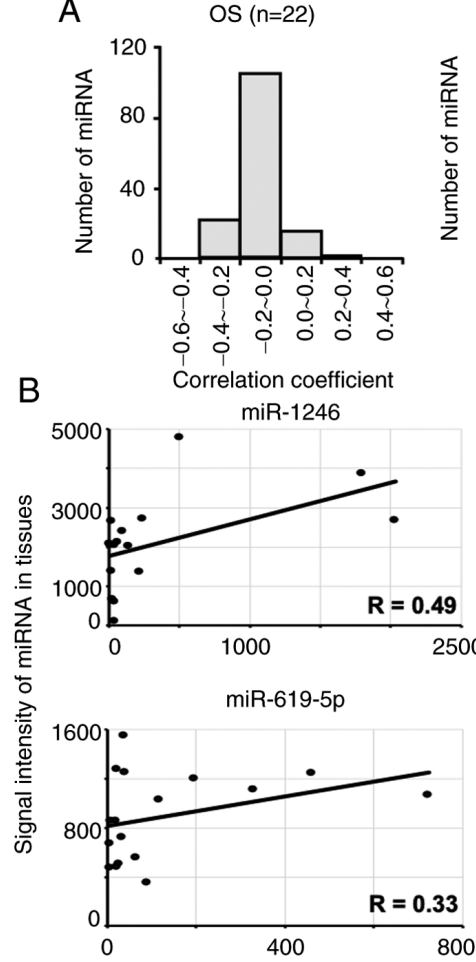

DDLPS $(n=17)$

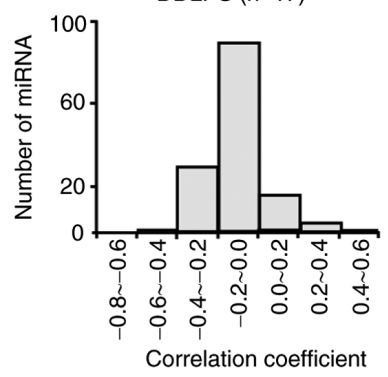

miR-4532
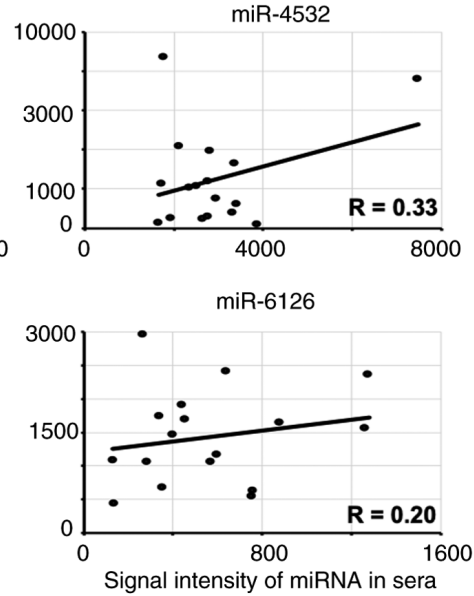

EWS ( $n=3)$

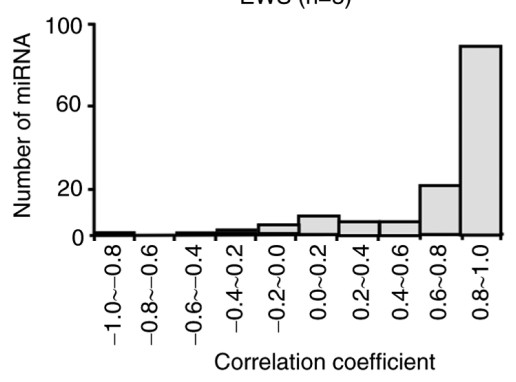

miR-4454

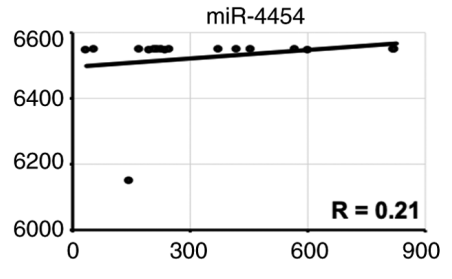

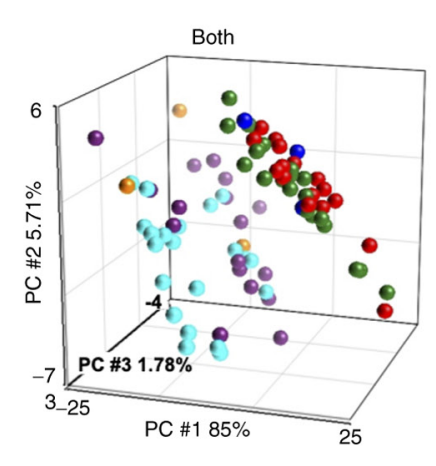

Tissue_DDLPS

Tissue_EWS

- Tissue_OS

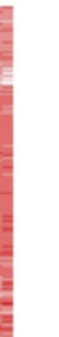

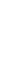

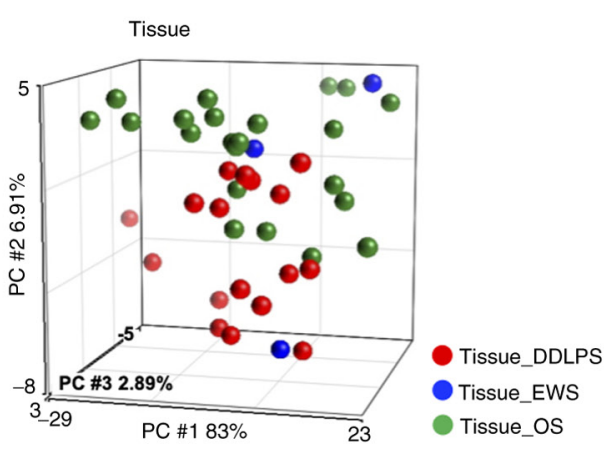


A

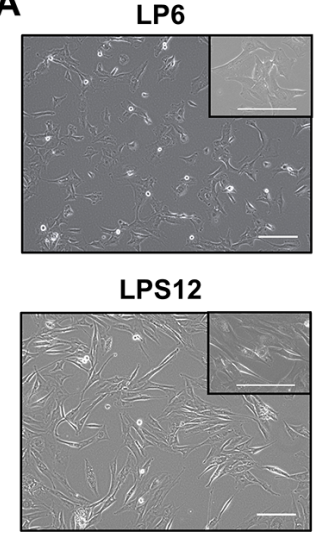

C
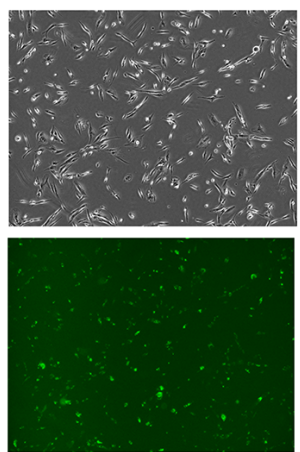

B

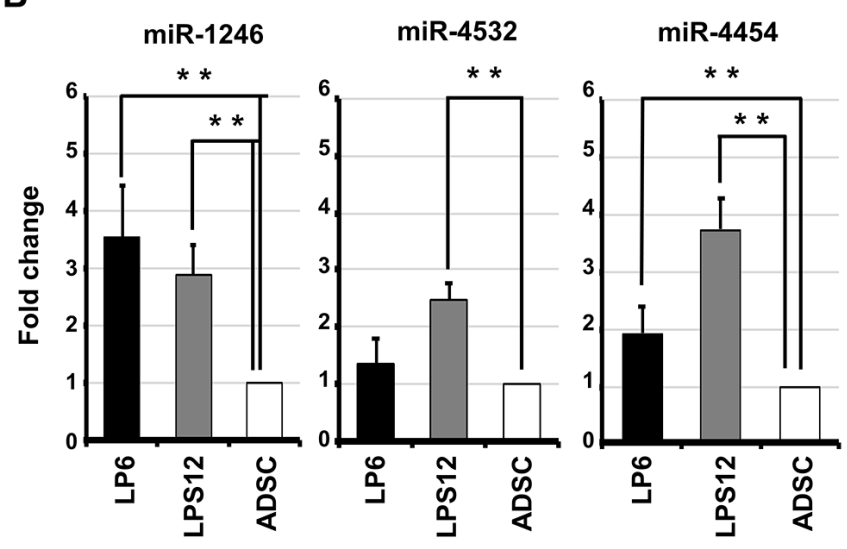

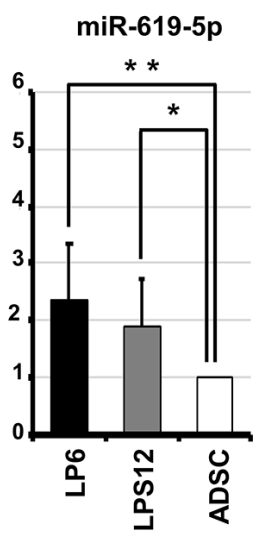

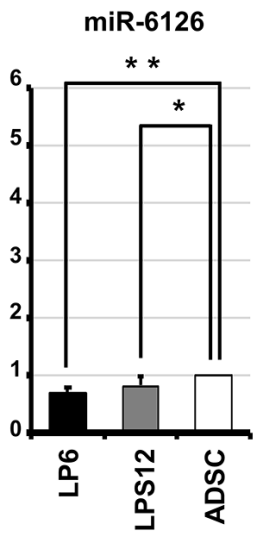

D
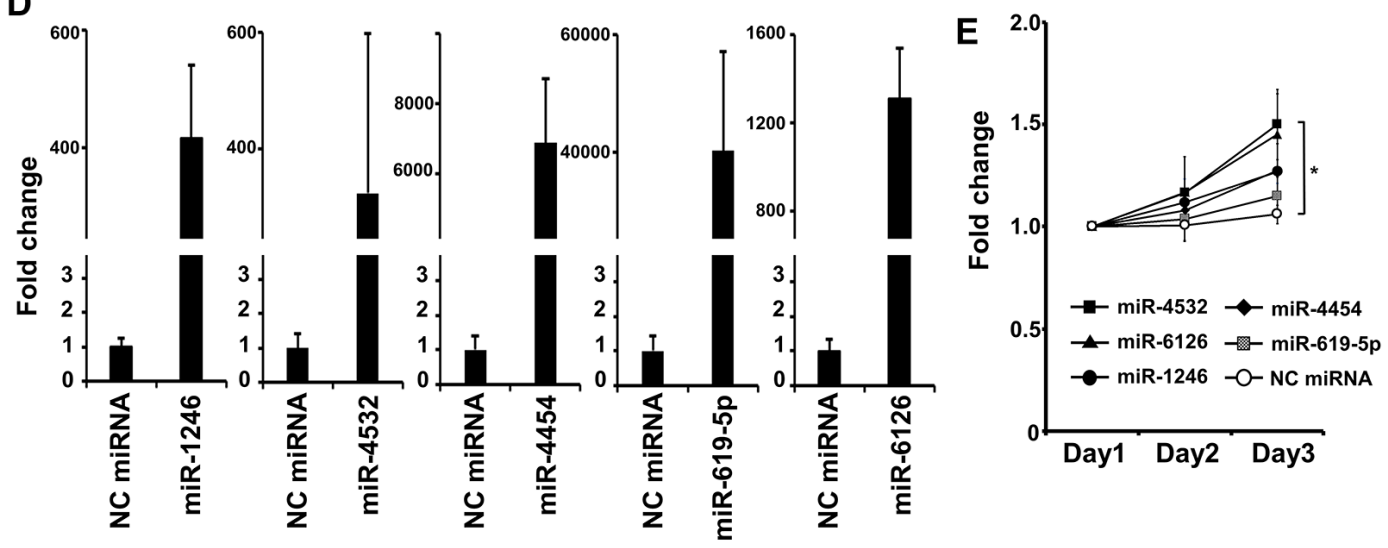

Figure 3. Expression of selected miRNAs in DDLPS cell lines and effect on cell proliferation. (A) Representative images of DDLPS cell lines. Scale bar, $200 \mu \mathrm{m}$. (B) Expression levels of each miRNA in LP6 and LPS12 cells normalized to that of ADSCs. (C) Transfection rate estimation using HiLyte Fluor488-labelled NC miRNA mimic in LP6 cells. Upper: Phase contrast; lower: Fluorescent image. Magnification, x40. (D) Reverse transcription quantitative-PCR analysis of miRNAs after miRNA mimic transfection. Fold-changes was calculated by normalizing miRNA levels to the NC values. The value of negative control set to 1.0. (E) Cell proliferation assay using LP6 cells. Each miRNA was transfected to LP6 cells. " $\mathrm{P}<0.05$ and ${ }^{* *} \mathrm{P}<0.01$. NC, negative control; $\mathrm{miRNA} / \mathrm{miR}$, microRNA. ADSC, adipose-derived stem cells.

levels, the number of detected miRNAs was too low $(n=3)$ to determine statistical significance. However, several miRNAs in DDLPS were expressed at relatively high levels, including miR-1246, -4532, -4454, -619-5p and -6126. The correlation these miRNAs in tissues and sera in DDLPS is presented in Fig. 2B. The expression levels of certain miRNAs, such as $-1246,-4532$ and $-619-5 p$, were weakly correlated between serum and tissue samples $(\mathrm{R}=0.33-0.49)$, suggesting that these serum miRNAs could be derived from tumors in patients with DDLPS.

miRNA expression in DDLPS cell lines and effect on cell proliferation. It was examined whether the identified miRNAs were released from the human DDLPS cell lines LP6 and LPS12 (Fig. 3A). For measurement of the expression levels of the miRNAs in DDLPS cells, total RNA was extracted from LP6 and LPS12 cells and ADSCs as controls and subjected to quantitative PCR. The expression of miR-1246, -4532, -4454 and -619-5p was higher in LP6 and LPS12 cells compared with in ADSCs (Fig. 3B). The ratio of the expression level of miR-1246 was $3.52 \pm 0.89(\mathrm{P}<0.01)$ in LP6 and $2.87 \pm 0.52$ $(\mathrm{P}<0.01)$ in LPS12 cells. That of miR-4532 was $1.36 \pm 0.43$ $(\mathrm{P}=0.29)$ in LP6 and $2.48 \pm 0.28(\mathrm{P}<0.01)$ in LPS12 cells. That of miR-4454 was $1.90 \pm 0.47(\mathrm{P}<0.01)$ in LP6 and $3.72 \pm 0.52$
$(\mathrm{P}<0.01)$ in LPS12 cells. That of miR-619-5p was $2.36 \pm 0.99$ $(\mathrm{P}<0.01)$ in LP6 and $1.88 \pm 0.84(\mathrm{P}=0.02)$ in LPS12 cells. That of miR-6126 was $0.62 \pm 0.12(\mathrm{P}<0.01)$ in LP6 and $0.83 \pm 0.15$ $(\mathrm{P}=0.03)$ in LPS12. To examine the effects of these miRNAs on DDLPS cell proliferation, the transfection efficiency of miRNA in LP6 cells was examined using a fluorescently-labeled miRNA mimic negative control. Based on the fluorescence rate, efficiency was $92.5 \pm 3.3 \%$ (Fig. 3C). Then, LP6 cells were transiently transfected with miRNA mimics, and the overexpression of each miRNA was confirmed using RT-qPCR (Fig. 3D). The cell proliferation rates were monitored on days 1, 2 and 3 after transfection (Fig. 3E). The cells transfected with the miRNAs exhibited a higher proliferative capacity compared with the negative control. miR-4532 had a significant effect on promoting cell proliferation in LP6 cells $(\mathrm{P}<0.05)$. miR-1246, -4454 and $-619-5 p$ were significantly highly expressed in both DDLPS cell lines. This result indicated the possibility that these miRNAs are also highly expressed in DDLPS tissues.

DDLPS cells release exosomes containing specific miRNAs. Exosomes were isolated from LP6 cells, LPS12 cells and ADSCs by ultracentrifugation. Isolated exosomes were confirmed using the NanoSight system (Fig. 4A). Typical 
A
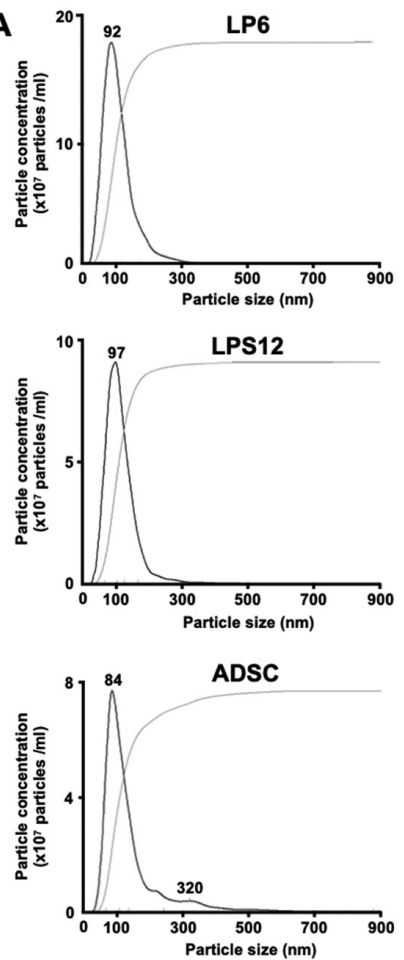

B

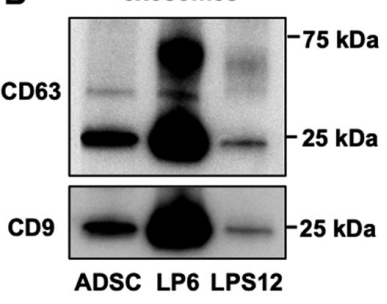

$1 \mu \mathrm{g}$ of exosomes / lane
C

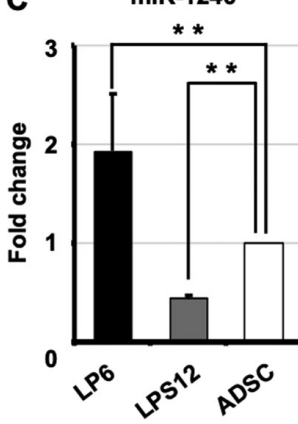

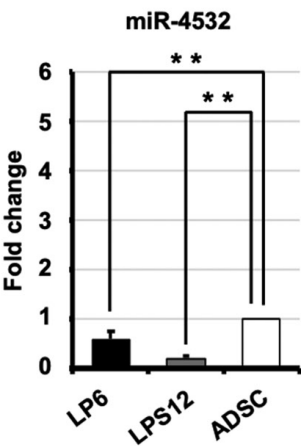

$\operatorname{miR}-6126$
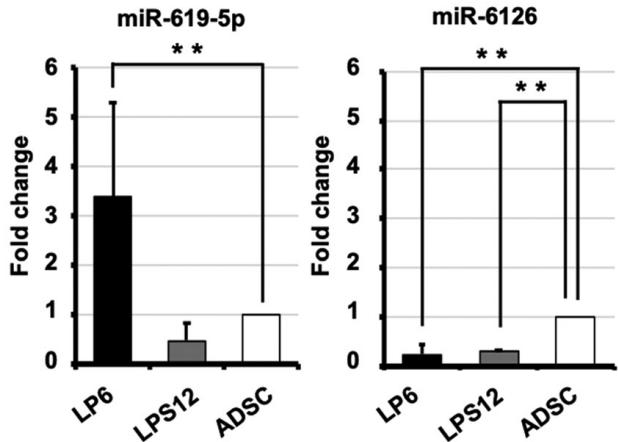

Figure 4. Detection of selected miRNAs in the exosomes of DDLPS cells. (A) Size evaluation of collected exosomes using the Nano tracking system. Number at the vertex of the graph indicates the particle size. (B) Immunoblotting for CD63 and CD9 in LP6 and LPS12 cells and ADSC. (C) Expression of each miRNA in exosomes from LP6 and LPS12 cells was normalized to that of ADSCs. ${ }^{*} \mathrm{P}<0.05$ and ${ }^{* *} \mathrm{P}<0.01$. miRNA/miR, microRNA; ADSC, adipose-derived stem cells.

exosome markers were confirmed with immunoblotting (Fig. 4B). CD63 and CD9 were expressed in the exosomes from LP6, LPS12 and ADSCs. Also, we previously confirmed the isolation of exosomes from ADSC by transmission electron microscopy (27). Total RNA was extracted from exosomes, and the expression levels of miRNAs were examined using RT-qPCR (Fig. 4C). miRNA expression levels were lower in exosomes from LPS12 cells compared with those from LP6 cells. The results of RT-qPCR analysis showed that miR-1246, -4454 and $-619-5 p$ were highly expressed in LP6 exosomes. The ratio of the expression level of exosomal miR-1246 was $1.92 \pm 0.89(\mathrm{P}<0.01)$ in LP6 and $0.42 \pm 0.52(\mathrm{P}<0.01)$ in LPS12 cells. That of miR-4532 was $0.56 \pm 1.91(\mathrm{P}<0.01)$ in LP6 and $0.18 \pm 0.37(\mathrm{P}<0.01)$ in LPS12 cells. That of miR-4454 was $8.35 \pm 0.16(\mathrm{P}<0.01)$ in LP6 and $0.28 \pm 0.03(\mathrm{P}=0.02)$ in LPS12 cells. That of miR-619-5p was $3.36 \pm 0.58(\mathrm{P}<0.01)$ in LP6 and $0.46 \pm 0.04(\mathrm{P}=0.67)$ in LPS12 cells. That of miR-6126 was $0.23 \pm 0.88(\mathrm{P}<0.01)$ in LP6 and $0.28 \pm 0.02(\mathrm{P}<0.01)$ in LPS1 2 cells. These results indicated that miR-1246, -4454 and $-619-5 p$ detected in the serum were derived from sarcoma tissues. Exosomal miR-1246, -4454 and -619-5p were detected in LP6 cells. This result indicated the possibility that these miRNAs are present in DDLPS serum.

\section{Discussion}

The use of multidrug chemotherapy in combination with surgery strongly improves the outcomes of patients with OS, resulting in a 5-year overall survival rate of $\sim 64-77 \%$ globally (28-31). However, the 5-year survival rate of patients with OS with metastatic disease and relapse at the first visit is $<20 \%$ globally $(1,32)$. The mortality rate of DDLPS is $\sim 60 \%$ globally, which can be partly attributed to tumors that are too large to be resected at the time of diagnosis (33). The present study identified miRNAs with potential value as biomarkers for DDLPS. A correlation between the serum and tissue expression of specific miRNAs [miR-1246 ( $\mathrm{R}=0.49)$, miR-619-5p $(\mathrm{R}=0.33)$, miR-4532 $(\mathrm{R}=0.33)$, miR-4454 $(\mathrm{R}=0.21)$ and miR-6126 $(\mathrm{R}=0.20)]$ and measured the expression levels of these miRNAs in DDLPS cell lines (LP6 and LPS12) and released exosomes.

Most of the miRNAs showing high levels of expression in the serum were also highly expressed in tissues in the present study, suggesting that serum miRNAs could be derived from tumor tissues. Although the number of EWS samples was too small to draw firm conclusions, the number of OS and DDLPS samples was considered to be sufficient for the analysis. There were differences in the miRNA expression between serum and tissues. When these miRNAs were evaluated individually, miR-1246 showed the highest correlation coefficient. To determine whether miRNAs were secreted from DDLPS cells, the miRNA expression levels between cells and exosomes were compared. miR-1246,-4454 and -619-5p were highly expressed in both DDLPS cell lines. miR-1246, -4454 and -619-5p were highly expressed in LP6 exosomes, even though the expression levels of miRNAs were generally low in LPS12 cells. The different results of these two cell lines may be because the cell line was isolated from part of one sample and may not have the characteristics of the original tumor. These data indicated that miR-1246, -4454 and -619-5p may be candidate miRNAs for the diagnosis of DDLPS. However, these miRNAs were not candidates for other LPS subtypes (well differentiated, myxoid/round cell or pleomorphic liposarcomas). 
There are few studies on miRNA biomarkers of sarcomas, but several studies have reported candidates for OS biomarkers (10-13,34). miR-195-5p, -199a-3p, -320a and $-374 a-5 p$ were significantly increased in the plasma of patients with OS compared with healthy controls (34). The expression of these four miRNAs decreased after tumor resections which were 83 extremities and 7 trunks and the expression of miR-195-5p and -199a-3p was significantly increased in patients with metastasis (34). miR-9 and -21 are upregulated in the blood samples of patients with OS, but -195, -Let7A, $-199 a-3 p$ and -143 are downregulated (10-13). These studies differed from the present report in that they compared blood samples of patients with OS with those of healthy controls. Moreover, Fujiwara et al (35) identified miR-25-3p as a diagnostic and prognostic biomarker of OS in vitro, in vivo and in clinical samples.

miR-155 was previously reported as a biomarker for the diagnosis of DDLPS. miR-155 is highly expressed in the tissues and plasma (36). Similarly, the high expression of miR-155 in tissues is correlated with poor prognosis (18). The present study examined the correlation between miRNA expression in the serum and tissue and serum miRNA levels may be used for predicting patient with DDLPS prognosis.

The present assessment of the effect of miRNAs on cell proliferation showed that miR-4532 and -6126 significantly promoted DDLPS cell proliferation. This finding suggested that these miRNAs may promote the progression of DDLPS. Meanwhile, other papers have reported that miR-4532 promotes tumor progression and that miR-6126 suppresses tumor progression. Breast cancer cells overexpressing miR-4532 have enhanced cell viability upon administration of adriamycin (37). miR-4532 targeting hypermethylation in cancer 1 (HIC-1), and overexpression of HIC-1 in breast cancer cells suppresses cell invasion in Transwell assays (37). Conversely, enhanced expression of miR-6126 suppresses ovarian cancer progression. Ovarian cancer cells transfected with miR-6126 mimic have decreased cell migration and invasion (38). The reason for the discrepancy between these previous studies and the present results is the different cancer types. In addition, the present evaluation was insufficient because only cell proliferation was evaluated. Additional experiments are required to identify direct targets of miR-4532 and miR-6126 in DDLPS.

The present study identified specific miRNAs that were highly expressed in both the serum and tissues from patients with DDLPS, and in vitro experiments suggested that certain miRNAs were secreted from DDLPS cells. Taken together, the present results suggested that the identified miRNAs could be of value as biomarkers in DDLPS.

\section{Acknowledgements}

The authors would like to thank Ms Tomomi Fukuda (Division of Molecular and Cellular Medicine, National Cancer Center Research Institute), Dr Hiroko Tadokoro (Division of Molecular and Cellular Medicine, National Cancer Center Research Institute), Mr Tatsuya Suzuki (Division of Molecular and Cellular Medicine, National Cancer Center Research Institute), Ms Makiko Ichikawa (New Frontiers Research Institute, Toray Industries), Mr Junpei Kawauchi (New Frontiers Research Institute, Toray Industries) and
Mr Satoshi Kondou (New Frontiers Research Institute, Toray Industries) for technical assistance. The authors thank Ms Noriko Abe (Clinical Laboratory, National Cancer Center Research Institute) and Ms Michiko Ohori (Department of Biobank and Tissue Resources, National Cancer Center Research Institute) for collecting samples from the freezing room and Dr Kazuki Sudo (Department of Breast and Medical Oncology, National Cancer Center Hospital) for independent confirmation of participant eligibility. The authors thank Dr Hitoshi Ichikawa (Department of Clinical Genomics, National Cancer Center Research Institute) for mediating the transfer of DDLPS cells.

\section{Funding}

The study was funded by The National Cancer Center Research and Development Fund (grant no. 29-A-1) and through a Development of Diagnostic Technology for Detection of miRNA in Body Fluids grant from the Japan Agency for Medical Research and Development.

\section{Availability of data and materials}

All data generated or analyzed during this study are included in this published article.

\section{Authors' contributions}

IK, NA, JM, YY, TY and RT performed the experiments, analyzed data and wrote the manuscript. EK, HC, AK and TO conceived the study, and analyzed and interpreted the data. ST, HS, KK and HF contributed to data analysis. IK, JM and YY confirmed the authenticity of raw data. All authors read and approved the final manuscript.

\section{Ethics approval and consent to participate}

The study was approved by The National Cancer Centre Hospital Institutional Review Board (Tokyo, Japan; approval nos. 2004-050, 2013-111 and 2015-266). The reason for several approval numbers was that the data from several studies used for reanalysis in the present study. Written informed consent was obtained from each participant. When the patient was under 20 years old, the informed consent was obtained from their parents, relatives or guardians of minors.

\section{Patient consent for publication}

Not applicable.

\section{Competing interests}

The authors declare that they have no competing interests.

\section{References}

1. Fletcher CDM, Bridge JA, Hogendoorn PCW and Mertens F: WHO classification of soft tissue tumours. 4th edition. IARC, Lyon, 2013.

2. Ottaviani G and Jaffe N: The epidemiology of osteosarcoma. Cancer Treat Res 152: 3-13, 2009. 
3. Kansara M, Teng MW, Smyth MJ and Thomas DM: Translational biology of osteosarcoma. Nat Rev Cancer 14: 722-735, 2014.

4. Mack TM: Sarcomas and other malignancies of soft tissue, retroperitoneum, peritoneum, pleura, heart, mediastinum, and spleen. Cancer 75 (Suppl 1): S211-S244, 1995.

5. Dalal KM, Kattan MW, Antonescu CR, Brennan MF and Singer S: Subtype specific prognostic nomogram for patients with primary liposarcoma of the retroperitoneum, extremity, or trunk. Ann Surg 244: 381-391, 2006.

6. Lin S and Gregory RI: MicroRNA biogenesis pathways in cancer. Nat Rev Cancer 15: 321-333, 2015.

7. Ling H, Fabbri $M$ and Calin GA: MicroRNAs and other non-coding RNAs as targets for anticancer drug development. Nat Rev Drug Discov 12: 847-865, 2013.

8. Xu R, Rai A, Chen M, Suwakulsiri W, Greening DW and Simpson RJ: Extracellular vesicles in cancer-implications for future improvements in cancer care. Nat Rev Clin Oncol 15: 617-638, 2018

9. Hoshino A, Costa-Silva B, Shen TL, Rodrigues G, Hashimoto A, Tesic Mark M, Molina H, Kohsaka S, Di Giannatale A, Ceder S, et al: Tumour exosome integrins determine organotropic metastasis. Nature 527: 329-35, 2015.

10. Cai H, Zhao H, Tang J and Wu H: Serum miR-195 is a diagnostic and prognostic marker for osteosarcoma. J Surg Res 194 505-510, 2015.

11. Hua J, Liu D, Cao L, Wang D, Wu T, Lin F, Su P, Niu Y and Sun Y: Diagnostic and prognostic values of blood microRNA-Let7A for osteosarcoma. J Bone Oncol 12: 65-68, 2018

12. Fei D, Li Y, Zhao D, Zhao K, Dai L and Gao Z: Serum miR-9 as a prognostic biomarker in patients with osteosarcoma. J Int Med Res 42: 932-937, 2014

13. Ouyang L, Liu P, Yang S, Ye S, Xu W and Liu X: A three-plasma miRNA signature serves as novel biomarkers for osteosarcoma. Med Oncol 30: 340, 2013.

14. Xu JF, Wang YP, Zhang SJ, Chen Y, Gu HF, Dou XF, Xia B, Bi Q and Fan SW: Exosomes containing differential expression of microRNA and mRNA in osteosarcoma that can predict response to chemotherapy. Oncotarget 8: 75968-75978, 2017.

15. Vos M, Boeve WC, van Ginhoven TM, Sleijfer S, Verhoef C and Grünhagen DJ: Impact of primary tumor location on outcome of liposarcoma patients, a retrospective cohort study. Eur J Surg Oncol 45: 2437-2442, 2019.

16. Jour G, Gullet A, Liu M and Hoch BL: Prognostic relevance of Fédération Nationale des Centres de Lutte Contre le Cancer grade and MDM2 amplification levels in dedifferentiated liposarcoma: A study of 50 cases. Mod Pathol 28: 37-47, 2015

17. Vincenzi B, Iuliani M, Zoccoli A, Pantano F, Fioramonti M De Lisi D, Frezza AM, Rabitti C, Perrone G, Onetti Muda A, et al: Deregulation of dicer and mir-155 expression in liposarcoma. Oncotarget 6: 10586-10591, 2015.

18. Kapodistrias N, Mavridis K, Batistatou A, Gogou P, Karavasilis V, Sainis I, Briasoulis E and Scorilas A: Assessing the clinical value of microRNAs in formalin-fixed paraffin-embedded liposarcoma tissues: Overexpressed miR-155 is an indicator of poor prognosis. Oncotarget 8: 6896-6913, 2017.

19. Lee DH, Amanat S, Goff C, Weiss LM, Said JW, Doan NB, Sato-Otsubo A, Ogawa S, Forscher C and Koeffler HP Overexpression of miR-26a-2 in human liposarcoma is correlated with poor patient survival. Oncogenesis 2: e47, 2013.

20. Casadei L, Calore F, Creighton CJ, Guescini M, Batte K, Iwenofu OH, Zewdu A, Braggio DA, Bill KL, Fadda P, et al: Exosome-derived miR-25-3p and miR-92a-3p stimulate liposarcoma progression. Cancer Res 77: 3846-3856, 2017.

21. Asano N, Matsuzaki J, Ichikawa M, Kawauchi J, Takizawa S, Aoki Y, Sakamoto H, Yoshida A, Kobayashi E, Tanzawa Y, et al: A serum microRNA classifier for the diagnosis of sarcomas of various histological subtypes. Nat Commun 10: 1299, 2019.

22. Usuba W, Urabe F, Yamamoto Y, Matsuzaki J, Sasaki H, Ichikawa M, Takizawa S, Aoki Y, Niida S, Kato K, et al: Circulating miRNA panels for specific and early detection in bladder cancer. Cancer Sci 110: 408-419, 2019.
23. Yokoi A, Matsuzaki J, Yamamoto Y, Yoneoka Y, Takahashi K, Shimizu H, Uehara T, Ishikawa M, Ikeda SI, Sonoda T, et al: Integrated extracellular microRNA profiling for ovarian cancer screening. Nat Commun 9: 4319, 2018.

24. Yokoi A, Yoshioka Y, Yamamoto Y, Ishikawa M, Ikeda SI, Kato T, Kiyono T, Takeshita F, Kajiyama H, Kikkawa F and Ochiya T: Malignant extracellular vesicles carrying MMP1 mRNA facilitate peritoneal dissemination in ovarian cancer. Nat Commun 8: 14470, 2017

25. Livak KJ and Schmittgen TD: Analysis of relative gene expression data using real-time quantitative PCR and the 2(-Delta Delta $\mathrm{C}(\mathrm{T})$ ) method. Methods 25: 402-408, 2001

26. Yoshioka Y, Konishi Y, Kosaka N, Katsuda T, Kato T and Ochiya T: Comparative marker analysis of extracellular vesicles in different human cancer types. J Extracell Vesicles 18: 2, 2013.

27. Zhou Y, Yamamoto Y, Takeshita F, Yamamoto T, Xiao Z and Ochiya T: Delivery of miR-424-5p via extracellular vesicles promotes the apoptosis of MDA-MB-231 TNBC cells in the tumor microenvironment. Int J Mol Sci 22: 844, 2021.

28. Iwamoto Y, Tanaka K, Isu K, Kawai A, Tatezaki S, Ishii T, Kushida K, Beppu Y, Usui M, Tateishi A, et al: Multiinstitutional phase II study of neoadjuvant chemotherapy for osteosarcoma (NECO study) in Japan: NECO-93J and NECO-95J. J Orthop Sci 14: 397-404, 2009.

29. Bacci G, Bertoni F, Longhi A, Ferrari S, Forni C, Biagini R, Bacchini P, Donati D, Manfrini M, Bernini G and Lari S: Neoadjuvant chemotherapy for high-grade central osteosarcoma of the extremity. Histologic response to preoperative chemotherapy correlates with histologic subtype of the tumor. Cancer 97: 3068-3075, 2003.

30. Bacci G, Briccoli A, Ferrari S, Longhi A, Mercuri M, Capanna R, Donati D, Lari S, Forni C and DePaolis M: Neoadjuvant chemotherapy for osteosarcoma of the extremity: Long-term results of the Rizzoli's 4th protocol. Eur J Cancer 37: 2030-2039, 2001.

31. Bajpai J, Chandrasekharan A, Talreja V, Simha V, Chandrakanth MV, Rekhi B, Khurana S, Khan A, Vora T, Ghosh J, et al: Outcomes in non-metastatic treatment naive extremity osteosarcoma patients treated with a novel non-high dosemethotrexate-based, dose-dense combination chemotherapy regimen 'OGS-12'. Eur J Cancer 85: 49-58, 2017.

32. Chou AJ, Geller DS and Gorlick R: Therapy for osteosarcoma: Where do we go from here? Paediatr Drugs 10: 315-27, 2008.

33. Henricks WH, Chu YC, Goldblum JR and Weiss SW: Dedifferentiated liposarcoma: A clinicopathological analysis of 155 cases with a proposal for an expanded definition of dedifferentiation. Am J Surg Pathol 21: 271-281, 1997.

34. Lian F, Cui Y, Zhou C, Gao K and Wu L: Identification of a plasma four-microRNA panel as potential noninvasive biomarker for osteosarcoma. PLoS One 10: e0121499, 2015.

35. Fujiwara T, Uotani K, Yoshida A, Morita T, Nezu Y, Kobayashi E, Yoshida A, Uehara T, Omori T, Sugiu K, et al: Clinical significance of circulating miR-25-3p as a novel diagnostic and prognostic biomarker in osteosarcoma. Oncotarget 8: 33375-33392, 2017.

36. Boro A, Bauer D, Born W and Fuchs B: Plasma levels of miRNA-155 as a powerful diagnostic marker for dedifferentiated liposarcoma. Am J Cancer Res 6: 544-552, 2016.

37. Feng F, Zhu X, Wang C, Chen L, Cao W, Liu Y, Chen Q and $\mathrm{Xu}$ W: Downregulation of hypermethylated in cancer- 1 by miR-4532 promotes adriamycin resistance in breast cancer cells. Cancer Cell Int 18: 127, 2018.

38. Kanlikilicer P, Rashed MH, Bayraktar R, Mitra R, Ivan C, Aslan B,Zhang X, Filant J, Silva AM, Rodriguez-Aguayo C, et al: Ubiquitous release of exosomal tumor suppressor miR-6126 from ovarian cancer cells. Cancer Res 76: 7194-7207, 2016.

This work is licensed under a Creative Commons Attribution-NonCommercial-NoDerivatives 4.0 International (CC BY-NC-ND 4.0) License. 